\title{
HISTOLOGICAL CHORIOAMNIONITIS IS NOT ASSOCIATED WITH COGNITIVE OUTCOME AT 5 YEARS IN LOW BIRTH WEIGHT CHILDREN
}

\author{
P.I. Kaaresen ${ }^{1,2}$, C. Klingenberg ${ }^{1,2}$, T.A. Hanssen ${ }^{3}$, J.A. Rønning ${ }^{1,2}$, S.E. Ulvund ${ }^{4}$, L.B. Dahl ${ }^{1,2}$ \\ ${ }^{1}$ Pediatric Department, University Hospital of North Norway, ${ }^{2}$ Department of Clinical Medine, University of \\ Tromsø, ${ }^{3}$ Department of Pathology, University Hospital of North Norway, Tromsø, ${ }^{4}$ Institute for Educational \\ Research, University of Oslo, Oslo, Norway
}

Background and aims: Prenatal inflammation and the fetal inflammatory response have been associated with preterm birth and subsequent neonatal lung and brain disorders. However, there is a paucity of information about the relation between prenatal infections and cognitive outcome in preterm children. The aim of this study was to examine the effect of prenatal inflammation on cognitive outcome at 5 years corrected age in low birthweight children.

Methods: Placentas from mothers who gave birth to an infant with birth weight $<2000 \mathrm{~g}$ and participated in a randomised controlled trial of an early intervention program were examined regarding the presence of histological chorioamnionitis (HCA). At 5 years corrected age cognitive outcomes was assessed with Wechslers Preschool and Primary Scale of Intelligence-Revised (WPPSI).

Results: A total of 124 infants were included. Mean birth weight was $1397 \mathrm{~g}$ (SD 409) and mean gestational age $30.1 \mathrm{w}$ (SD 3.1). Placental histology showed no signs of HCA in $42(34 \%)$, mild HCA in $62(50 \%)$ and severe HCA in $20(16 \%)$ of the placentas. WPPSI scores were available for 113 children. There were no significant differences between the HCA groups in WPPSI verbal, performance or full-scale IQ adjusted for gestational age and possible intervention effect.

Conclusion: HCA is not independently associated with cognitive outcomes at 5 years in low birth weight children. 